\title{
Colorimetric RT-LAMP and LAMP-sequencing for \\ Detecting SARS-CoV-2 RNA in Clinical Samples
}

Konrad Herbst ${ }^{1, *}$, Matthias Meurer ${ }^{1}$, Daniel Kirrmaier ${ }^{2}$, Simon Anders ${ }^{1}$, Michael Knop ${ }^{1,2,3, *}$ and Viet Loan Dao Thi, *

${ }^{1}$ Center for Molecular Biology of Heidelberg University (ZMBH), Heidelberg, Germany; ${ }^{2}$ German Cancer Research Center (DKFZ), Heidelberg, Germany; ${ }^{3} \mathrm{DKFZ}-\mathrm{ZMBH}$ Alliance, Heidelberg, Germany; ${ }^{4}$ Schaller Research Group, Department of Infectious Diseases, Virology, Heidelberg University, Heidelberg, Germany

*For correspondence: $\quad$ k.herbst@zmbh.uni-heidelberg.de; $\quad$ m.knop@zmbh.uni-heidelberg.de; vietloan.daothi@med-uni.heidelberg.de

[Abstract] During pandemics, such as the one caused by SARS-CoV-2 coronavirus, simple methods to rapidly test large numbers of people are needed. As a faster and less resource-demanding alternative to detect viral RNA by conventional qPCR, we used reverse transcription loop-mediated isothermal amplification (RT-LAMP). We previously established colorimetric RT-LAMP assays on both purified and unpurified SARS-CoV-2 clinical specimens and further developed a multiplexed sequencing protocol (LAMP-sequencing) to analyze the outcome of many RT-LAMP reactions at the same time (Dao Thi et al., 2020). Extending on this work, we hereby provide step-by-step protocols for both RT-LAMP assays and read-outs.

Keywords: RT-LAMP, LAMP-sequencing, SARS-CoV-2 detection, Tn5 tagmentation, colorimetric assay

[Background] The new SARS-CoV-2 coronavirus poses a major public health problem (reviewed in Li et al., 2020). In the absence of efficient antiviral treatments and a protective vaccine, preventing local outbreaks by mass testing is critical. The standard diagnostic pipeline to detect SARS-CoV-2 infections is based on the isolation of viral RNA from clinical specimens, a reverse-transcription (RT) reaction to transcribe the RNA into CDNA, and detection by a semi-quantitative DNA polymerase chain reaction (qPCR) (Corman et al., 2020). Yet, commercial RNA isolation and RT-qPCR kits are costly, timeconsuming, and shortages of supplies during the pandemics limit high-throughput testing requiring alternative solutions (Klein et al., 2020).

In our recent study (Dao Thi et al., 2020), we used reverse transcription loop-mediated isothermal amplification (RT-LAMP) (Notomi et al., 2020) as an alternative to detect SARS-CoV-2 RNA in clinical specimens. We developed and characterized colorimetric RT-LAMP assays on both purified and unpurified pharyngeal swab specimens. We also developed a multiplexed sequencing protocol based on tagmentation for enzymatic addition of barcoded sequencing library adapters. This enables the analysis of many RT-LAMP reactions at the same time. Here, we present detailed step-by-step protocols to further facilitate the application of RT-LAMP for mass testing. 


\section{Materials and Reagents}

1. $1.5 \mathrm{ml}$ tubes (Eppendorf), room temperature

2. Filter tips (for pipettes and liquidator), room temperature

3. 96-well plate (Eppendorf, catalog number: 0030128672), room temperature

4. Nuclease-free water (Ambion, catalog number: AM9937), room temperature

5. Ethanol for Molecular Biology

6. WarmStart Colorimetric RT-LAMP 2× Master Mix (New England Biolabs, catalog number: M1800), $-20^{\circ} \mathrm{C}$

7. $10 \times$ primer mix for RT-LAMP assay as in Table 1 (Sigma-Aldrich), $-20^{\circ} \mathrm{C}$

Table 1. $\mathbf{N}$ gene primer for RT-LAMP assay. Primer sequences were designed by Zhang et al. (2020).

\begin{tabular}{|l|l|l|}
\hline Name & Sequence & $\begin{array}{l}\text { Concentration in } \\
\mathbf{1 0 x} \text { primer } \mathbf{m i x}(\boldsymbol{\mu} \mathbf{M})\end{array}$ \\
\hline GeneN-A-F3 & TGGCTACTACCGAAGAGCT & $\mathbf{2}$ \\
\hline GeneN-A-B3 & TGCAGCATTGTTAGCAGGAT & 2 \\
\hline GeneN-A-FIP & TCTGGCCCAGTTCCTAGGTAGTCCAGACGAATTCGTGGTGG & 16 \\
\hline GeneN-A-BIP & AGACGGCATCATATGGGTTGCACGGGTGCCAATGTGATCT & $\mathbf{1 6}$ \\
\hline GeneN-A-LF & GGACTGAGATCTTTCATTTTACCGT & 4 \\
\hline GeneN-A-LB & ACTGAGGGAGCCTTGAATACA & 4 \\
\hline
\end{tabular}

8. LAMP-sequencing primers as in Table 2 (Sigma-Aldrich), $-20^{\circ} \mathrm{C}$

Table 2. LAMP-sequencing primers. The full table is available as Table S4 in Dao Thi et al. (2020). [Phos] = phosphorylation, [SpcC3] = C3 spacer group, N, X, Y indicate one of the bases [GATC] ( $N$ are random bases while $X$ and $Y$ belong to respective inline barcodes used for multiplexing).

\begin{tabular}{|l|l|}
\hline Name & Sequence \\
\hline Tn5hY-Rd2-Wat-SC3 & [Phos ] CTGTCTCTTATACACATCT [ SpcC3] \\
\hline P5-UMl-xi5XXX-ME.fw & CGGCGACCACCGAGATCTACACNNNNNNNNNXXXXXXXXXXXCGTCGGCAGCG \\
\hline P5.fw & AATGATATGTGTATAAGAGACAG \\
\hline & GTCTCGTGGGCTCGGAGATGTGTATAAGAGACAGTGTATTCAAGGCTCCCTCAG \\
P7nxt-GeneN-A-LBrc & T \\
\hline P7-xi7YYY & CAAGCAGAAGACGGCATACGAGATYYYYYYYYYYYYTCTCGTGGGCTCGGAG \\
\hline
\end{tabular}

9. Optically clear adhesive seal (Kisker Biotech, catalog number: GK480-OS), room temperature

10. Adhesive aluminum foil seal (Steinbrenner Laborsysteme, catalog number: SL-AM0550), room temperature

11. Pierceable foil (Brooks Life Sciences, catalog number: 4ti-0566/96), room temperature Copyright (c) 2021 The Authors; exclusive licensee Bio-protocol LLC. 
12. $200 \mathrm{ng} / \mu \mathrm{l} \operatorname{Tn} 5$ (E54K, L372P) Transposase (purified according to Hennig et al., $2018,-80{ }^{\circ} \mathrm{C}$ )

13. $0.2 \%$ SDS solution (room temperature)

14. AMPureXP bead (Beckman Coulter, catalog number: A63881), $4{ }^{\circ} \mathrm{C}$

15. NEBNext Q5 HotStart polymerase (New England Biolabs, catalog number: M0543), $-20^{\circ} \mathrm{C}$

16. NucleoSpin Gel and PCR Clean-up mini kit (Macherey-Nagel, catalog number: 740609), room temperature

17. NEBNext Library Quant Kit for Illumina (New England Biolabs, catalog number: E7630), $-20^{\circ} \mathrm{C}$

18. [Tris(hydroxymethyl)methylamino]propanesulfonic acid (TAPS)

19. $\mathrm{MgCl}_{2}$

20. Dimethylformamide (DMF)

21. Freshly prepared $5 \times$ tagmentation buffer (see Recipes)

Note: All chemicals purchased from Sigma-Aldrich except when indicated otherwise.

\section{Equipment}

1. Pipetman $L P 2 L, 0.2-2 \mu$ (Gilead, catalog number: FA10001M)

2. Pipetman L P20L, 2-20 $\mu$ (Gilead, catalog number: FA10003M)

3. Pipetman L P200L, 20-200 $\mu \mathrm{l}$ (Gilead, catalog number: FA10005M)

4. Pipetman L P1000L, 100-1, $000 \mu \mathrm{l}$ (Gilead, catalog number: FA10006M)

5. Pipetman L Multichannel P8 $\times 20 \mathrm{~L}, 2-20 \mu \mathrm{l}$ (Gilead, catalog number: FA10009)

6. Liquidator 96 2-20 $\mu \mathrm{l}$ (Mettler Toledo, catalog number: LIQ-96-20)

7. Thermocycler (Biometra, TAdvanced $96 \mathrm{~S}$ )

8. Absorbance reader (Tecan, model: Infinite M200/Spark Cyto)

9. Centrifuge (Eppendorf, model: $5430 \mathrm{R}$ )

10. Table top centrifuge (Heraeus, model: Pico 21)

11. Magnetic stand (6 Tube Magnetic Stand; Ambion, catalog number: 10055)

12. NextSeq 550 machine (Illumina)

\section{Procedure}

A schematic diagram depicting the whole experimental procedure is shown in Figure 1. 


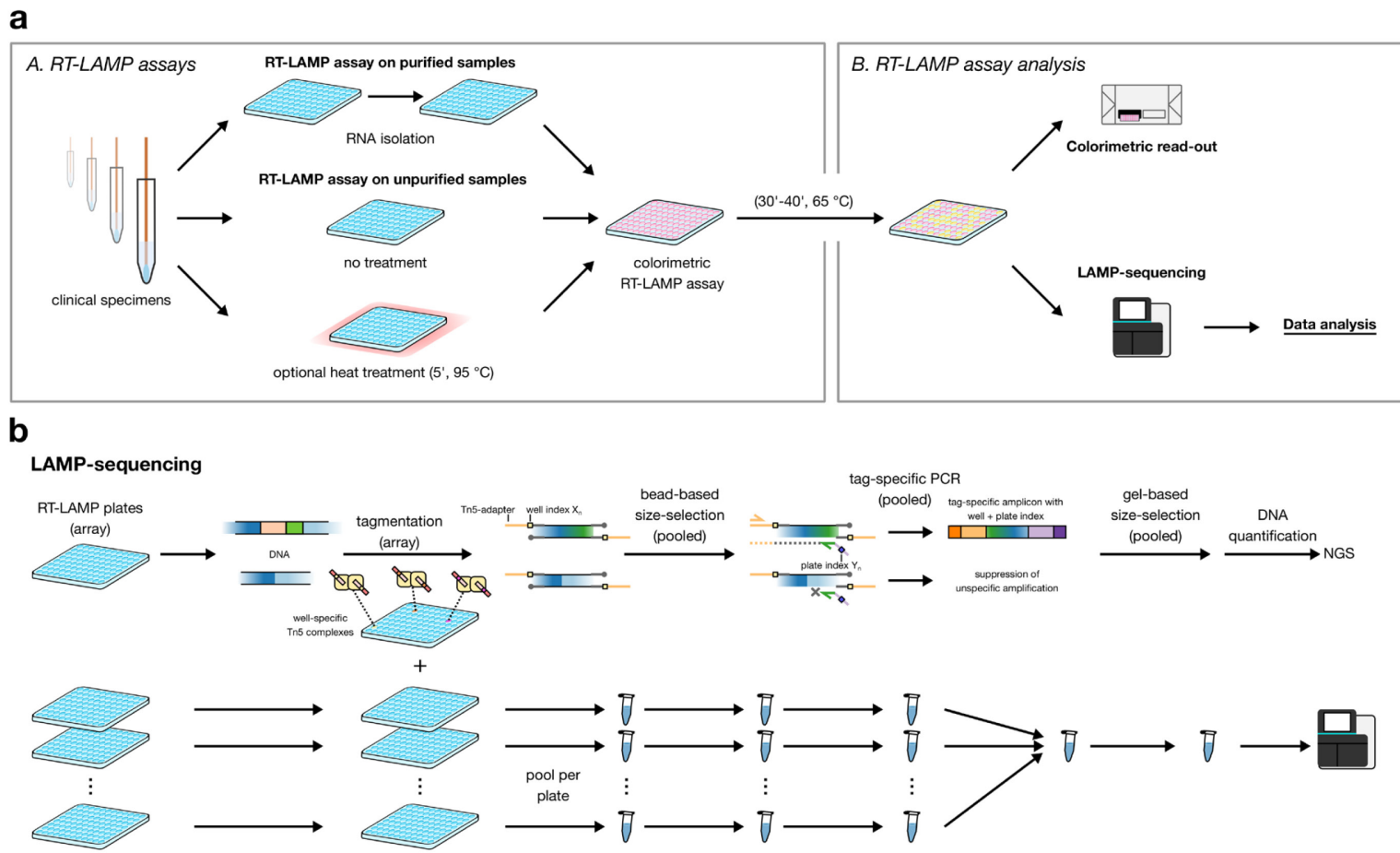

Figure 1. Overview of experimental procedures. (a) RT-LAMP assays can be performed with purified or unpurified clinical specimens and then analyzed using a colorimetric or LAMP-sequencing read-out.

(b) Flow-chart of LAMP-sequencing library preparation.

A. RT-LAMP assays

RT-LAMP assay on purified samples

1. Isolate RNA from clinical specimen according to manufacturer's protocol.

2. Assemble RT-LAMP master mix in a $1.5 \mathrm{ml}$ Eppendorf tube by adding $6.25 \mu \mathrm{l}$ of the $2 \times$ Master Mix, $1.25 \mu \mathrm{l}$ of $10 \times$ primer mix, and $4 \mu \mathrm{l}$ nuclease-free water per reaction.

3. Vortex and spin down.

4. Distribute $11.5 \mu \mathrm{l}$ of master mix into each well of a 96 -well plate using a multichannel pipette.

5. Add $1 \mu$ l of isolated RNA into wells with master mix.

6. Seal plate with optically clear adhesive seal.

7. Briefly spin down plate.

8. Incubate for $30 \mathrm{~min}$ at $65^{\circ} \mathrm{C}$ in a thermocycler (with the lid heated to $75^{\circ} \mathrm{C}$ ).

\section{RT-LAMP assay on unpurified samples}

1. For hot swab-to-RT-LAMP assays, pipette $50 \mu \mathrm{l}$ of clinical specimen into 96-well plate and seal with pierceable foil.

2. Heat up plate for $5 \mathrm{~min}$ at $95^{\circ} \mathrm{C}$ in 96 -well plate in a PCR cycler (with the lid heated to $105^{\circ} \mathrm{C}$ ).

3. Cool down, spin briefly, and keep plate on ice.

4. Assemble RT-LAMP master mix in a $1.5 \mathrm{ml}$ Eppendorf tube by adding $10 \mu \mathrm{l}$ of the $2 \times$ Master Mix, $2 \mu \mathrm{l}$ of $10 \times$ primer mix, and $7 \mu \mathrm{l}$ nuclease-free water per reaction. 
5. Vortex and spin down.

6. Distribute $19 \mu \mathrm{l}$ of master mix into each well of a 96-well plate using a multichannel pipette.

7. For direct swab-to-RT-LAMP assays, pipette $1 \mu \mathrm{l}$ of clinical specimen directly into wells with master mix.

8. For hot assays, pipette $1 \mu \mathrm{l}$ of prepared specimen (1-3) into wells with master mix.

9. Seal plate with optically clear adhesive seal.

10. Briefly spin down plate.

11. Incubate for $30 \mathrm{~min}$ at $65^{\circ} \mathrm{C}$ in a thermocycler (with the lid heated to $75^{\circ} \mathrm{C}$ ).

B. RT-LAMP assay analysis

\section{Colorimetric read-out}

1. Cool down 96 -well plate to $4{ }^{\circ} \mathrm{C}$ and spin down briefly.

2. Place 96-well plate into absorbance reader.

3. Measure absorbance at $434 \mathrm{~nm}$ and $560 \mathrm{~nm}$.

\section{LAMP-sequencing}

(All concentrations are given as final concentrations in reactions.)

1. Prepare transposon adapters by mixing individual barcoded adapter (P5-UMI-xi5XXX-ME.fw) with the primer Tn5hY-Rd2-Wat-SC3 at a final concentration of $25 \mu \mathrm{M}$ per primer in $5 \mu \mathrm{M}$ Tris$\mathrm{HCl}\left(\mathrm{pH}\right.$ 8.0) in a 96-well PCR plate using the Liquidator. Heat up to $99^{\circ} \mathrm{C}$ for $5 \mathrm{~min}$ and let the primers slowly anneal by cooling down to $20^{\circ} \mathrm{C}$ within 15 min using a thermocycler.

2. Mix transposase to a final concentration of $100 \mathrm{ng} / \mu \mathrm{l}$ with $1.25 \mu \mathrm{M}$ annealed adapters from step 1 in $50 \mathrm{mM}$ Tris- $\mathrm{HCl}(\mathrm{pH} 7.5)$ in 96-well PCR plates using the Liquidator. Assemble transposons by incubating the reaction for $1 \mathrm{~h}$ at $23^{\circ} \mathrm{C}$ in a thermocycler.

3. Freshly prepare the $5 \times$ tagmentation buffer according to the indicated composition.

4. Per well mix $1.2 \mu \mathrm{l}$ of the RT-LAMP product (equivalent to $\sim 200 \mathrm{ng}$ DNA) with $1.5 \mu \mathrm{l}$ of loaded transposase, $1.12 \mu \mathrm{l} 5 \times$ tagmentation buffer from step 3 and $1.8 \mu \mathrm{l}$ water to assemble the transposon reactions in 96-well PCR plates with the Liquidator. Incubate reactions at $55^{\circ} \mathrm{C}$ for $10 \mathrm{~min}$ in a thermocycler.

5. Stop the tagmentation reactions by adding $1.13 \mu \mathrm{l} 0.2 \%$ SDS per well and incubate for $10 \mathrm{~min}$ at room temperature. Pool the reactions into one single reaction each plate.

6. Perform size selection for fragments of approximately 300 to 600 bp by using the following twostep AMPure XP bead protocol (written for a pooled reaction from one plate).

a. Mix $50 \mu \mathrm{l}$ of pooled reaction with $50 \mu \mathrm{l}$ of water.

b. Remove large fragments by adding $55 \mu$ l of AMPure XP beads to the diluted samples. Mix by pipetting ten times and incubate at room temperature for $5 \mathrm{~min}$. Separate beads from supernatant by placing on a magnetic stand for $\sim 5 \mathrm{~min}$. Transfer the supernatant to a fresh eppendorf tube using a pipette without transferring beads.

c. Remove small fragments by adding $25 \mu \mathrm{l}$ of fresh beads to the supernatant. Mix by pipetting 
ten times and incubate at room temperature for $5 \mathrm{~min}$. Separate beads from supernatant by placing on a magnetic stand for $\sim 5$ min. Discard the supernatant containing the small fragments using a pipette without disturbing the bead pellet.

d. Wash DNA bound to beads by two washes with ethanol. For this, add $200 \mu \mathrm{l}$ ethanol (80\%) to the beads, mix by pipetting ten times and incubate at room temperature for $5 \mathrm{~min}$. Separate beads from ethanol by placing on a magnetic stand for $\sim 5$ min. Repeat this for a second wash. Let the beads air-dry for $10 \mathrm{~min}$.

e. Elute DNA from beads by adding $10 \mu \mathrm{l}$ of $5 \mathrm{mM}$ Tris- $\mathrm{HCl}(\mathrm{pH} 8.5)$, incubating for $5 \mathrm{~min}$ at room temperature and separating on a magnetic rack for $\sim 5 \mathrm{~min}$.

7. Perform one PCR reaction per plate using $1 \mu \mathrm{l}$ of size-selected eluate from step 6 as a template. Prepare PCR reactions with RT-LAMP-specific and Tn5-adapter-specific primers (P7nxtGeneN-A-LBrc and P7-xi7YYY, P5.fw) with the NEBNext Q5 HotStart polymerase according to the manufacturer's instruction. Use the following PCR conditions for amplification with a thermocycler: Two cycles at $62{ }^{\circ} \mathrm{C}$ for annealing and $90 \mathrm{~s}$ elongation, followed by two cycles at $65^{\circ} \mathrm{C}$ for annealing and $90 \mathrm{~s}$ elongation, and 13 cycles at $72^{\circ} \mathrm{C}$ annealing and $90 \mathrm{~s}$ elongation.

8. Pool all PCR reactions and perform a second size selection for fragments of approximately 400 to 550 bp: Run $20 \%$ of the pooled PCR reactions on a $2 \%$ agarose/Tris-acetate-EDTA gel, cut out the respective part of the lane and use a gel purification kit according to the manufacturer.

9. Quantify the library using for example a qPCR-based library quantification kit.

10. Perform a custom Illumina sequencing run on a NextSeq 550 machine based on the instructions of the manufacturer using $20 \%$ phiX spike-in and 136 cycles for the first read, 11 cycles to read the 11-nt-long plate index (i7) and 20 cycles to read the 11-nt-long well index (i5) and the 9-ntlong UMI.

\section{Data analysis}

A. Colorimetric RT-LAMP analysis

The results of the colorimetric RT-LAMP assay can be judged by naked eye. A clear color change from pink to orange or yellow is considered as SARS-CoV-2 positive after 30 min incubation at $65^{\circ} \mathrm{C}$. Color changes after $30 \mathrm{~min}$ can be caused by spurious amplification products and are therefore scored negative. For further validation, the RT-LAMP product can be analyzed by gel electrophoresis and should yield a distinct banding pattern as described previously (Dao Thi et al., 2020, see Figure 1 herein).

When the assay is analyzed by a plate reader, subtract absorbance reads $560 \mathrm{~nm}$ from $434 \mathrm{~nm}$ $(\triangle O D)$. An $\triangle O D$ value $>0.3$ is considered SARS-CoV-2 positive after 30 min incubation at $65^{\circ} \mathrm{C}$. For the hot swab-to-RT-LAMP assays, this read-out can be improved by subtracting the differences between the $\triangle O D$ values at time points $30 \mathrm{~min}$ and $10 \mathrm{~min}$ of the incubation at $65^{\circ} \mathrm{C}$. 


\section{B. LAMP-sequencing analysis}

Raw NGS results (single-end fastq file) need first to be converted to count tables using a workflow, which can be downloaded from GitHub (https://github.com/anders-biostat/LAMP-PaperFigures/tree/master/LAMP-sequencing_raw_read_processing). All the necessary software to run this workflow are summarized there. Individual processing steps can be run sequentially from inside this directory with the script '00-run_workflow.sh' used for illustration the example file 'LAMPsequencing_raw_sample100k.fastq.gz'. In order to run the workflow with a different dataset one needs to adapt the pathnames in '00-run_workflow.sh' accordingly. Two files are the result ('counts.tsv' and 'counts.Rda') which can be used for subsequent analysis. For example the count table to produce the respective figures for our RT-LAMP study (Dao Thi et al., 2020) is also present in this GitHub repository.

\section{$\underline{\text { Notes }}$}

All work with crude SARS-CoV-2 clinical specimens should be carried out in a biosafety level 2 cabinet until inactivation. We found that both purified and unpurified pharyngeal swab specimens as well as saliva specimens were compatible with RT-LAMP assays. Other types of specimens have to be tested.

In order to avoid contaminations and RNA degradation, all steps are carried out using filter tips and wearing gloves. In addition, keep clinical specimens on ice as much as possible to prevent RNA degradation. Master mix and test samples should be pipetted at different workplaces using different sets of pipettes. Ideally, the person executing the protocols has experience in molecular biology. Additional important considerations when using RT-LAMP reagents are listed in the Supplementary Material of our previously published work (Dao Thi et al., 2020).

\section{Recipes}

1. $5 \times$ tagmentation buffer

(Always prepare fresh.)

1 vol of 10x TAPS buffer (100 mM [Tris(hydroxymethyl)methylamino]propanesulfonic acid (TAPS) (pH 8.5), $50 \mathrm{mM} \mathrm{MgCl} 2$ )

1 vol of $100 \%(v / v)$ dimethylformamide (DMF)

\section{Acknowledgments}

This protocol was modified from our original method published previously (Dao Thi et al., 2020). K.H. was supported through a grant by the Deutsche Forschungsgemeinschaft (DFG; grant no. KN498/11-1) to M.K. V.L.D.T., was supported by the Chica and Heinz Schaller foundation. We would like to acknowledge the EMBL Protein Expression and Purification facility for production of the Tn5 
enzyme.

\section{Competing interests}

The authors declare no competing interests.

\section{$\underline{\text { References }}$}

1. Corman, V. M., Landt, O., Kaiser, M., Molenkamp, R., Meijer, A., Chu, D. K., Bleicker, T., Brunink, S., Schneider, J., Schmidt, M. L., Mulders, D. G., Haagmans, B. L., van der Veer, B., van den Brink, S., Wijsman, L., Goderski, G., Romette, J. L., Ellis, J., Zambon, M., Peiris, M., Goossens, H., Reusken, C., Koopmans, M. P. and Drosten, C. (2020). Detection of 2019 novel coronavirus (2019-nCoV) by real-time RT-PCR. Euro Surveill 25(3): 2000045.

2. Dao Thi, V. L., Herbst, K., Boerner, K., Meurer, M., Kremer, L. P., Kirrmaier, D., Freistaedter, A., Papagiannidis, D., Galmozzi, C., Stanifer, M. L., Boulant, S., Klein, S., Chlanda, P., Khalid, D., Barreto Miranda, I., Schnitzler, P., Krausslich, H. G., Knop, M. and Anders, S. (2020). A colorimetric RT-LAMP assay and LAMP-sequencing for detecting SARS-CoV-2 RNA in clinical samples. Sci Transl Med 12(556): eabc7075.

3. Hennig, B. P., Velten, L., Racke, I., Tu, C. S., Thoms, M., Rybin, V., Besir, H., Remans, K. and Steinmetz, L. M. (2018). Large-Scale Low-Cost NGS Library Preparation Using a Robust Tn5 Purification and Tagmentation Protocol. G3 (Bethesda) 8(1): 79-89.

4. Klein, S., Müller, T. G., Khalid, D., Sonntag-Buck, V., Heuser, A. M., Glass, B., Meurer, M., Morales, I., Schillak, A., Freistaedter, A., Ambiel, I., Winter, S. L., Zimmermann, L., Naumoska, T., Bubeck, F., Kirrmaier, D., Ullrich, S., Barreto Miranda, I., Anders, S., Grimm, D., Schnitzler, P., Knop, M., Kräusslich, H. G., Dao Thi, V. L., Börner, K. and Chlanda, P. (2020). SARS-CoV2 RNA Extraction Using Magnetic Beads for Rapid Large-Scale Testing by RT-qPCR and RTLAMP. Viruses 12(8): 863.

5. Li, H., Liu, L., Zhang, D., Xu, J., Dai, H., Tang, N., Su, X. and Cao, B. (2020). SARS-CoV-2 and viral sepsis: observations and hypotheses. Lancet 395(10235): 1517-1520.

6. Notomi, T., Okayama, H., Masubuchi, H., Yonekawa, T., Watanabe, K., Amino, N. and Hase, T. (2000). Loop-mediated isothermal amplification of DNA. Nucleic Acids Res 28(12): e63.

7. Zhang, Y., Odiwuor, N., Xiong, J., Sun, L., Nyaruaba, R. O., Wei, H. and Tanner, N. A. (2020). Rapid molecular detection of SARS-CoV-2 (COVID-19) virus RNA using colorimetric LAMP. medRxiv. https://doi.org/10.1101/2020.02.26.20028373. 